\title{
TOPOLOGICAL PROPERTIES OF SOUSLIN SUBSETS
}

\author{
BY
}

R. W. HANSELL

\begin{abstract}
Let $X$ be a subparacompact regular space such that the projection map $p: X \times \mathbf{P} \rightarrow X$, where $\mathbf{P}$ is the space of irrational numbers, preserves collections of sets having a $\sigma$-locally finite refinement. It is shown that $p$ then preserves generalized $F_{\sigma}$-sets. It follows that, if $X$ has any tpological property which is hereditary with respect to generalized $F_{\sigma}$-sets, then every Souslin subset of $X$ will also have this property in the relative topology. Such topological properties include nearly all covering properties (paracompactness, metacompactness, etc.), as well as normality, collectionwise normality, and the Lindelöf property. We show that the above mapping property will hold whenever $X$ is a $P(\omega)$-space, thus, in particular, when $X$ is any Souslin (hence any Baire) subset of a compact space crossed with a metrizable space. Additional topological properties of Souslin subsets, such as topological completeness, realcompactness, $z$-embeddedness, and the properties of being a $P$-space or $\Sigma$-space, are also considered.
\end{abstract}

1. Introduction. It is a well known fact that many (if not most) topological properties are hereditary with respect to $F_{\sigma}$-subsets. This is true, for example, with nearly all covering properties (such as paracompactness, metacompactness, etc.), and also for such familiar properties as normality, collectionwise normality, and the Lindelöf property. Standard examples can be used to show that one cannot, in general, expect these properties to be hereditary with respect to $F_{\sigma \delta}$-subsets (see 2.14 below). However, a fact that seems to be less well known, and one that is pivotal for the present paper, is that these properties continue to be hereditary with respect to the wider collection of "generalized $F_{\sigma}$-subsets" (see 2.1 below for the definition).

Here our aim is to investigate the problem of when such topological properties are hereditary with respect to all Souslin subsets (i.e., subsets generated from the closed sets via the $(A)$-operation of Souslin). Our results show, in particular, that this will hold true for the class of all subparacompact (or strong) $\Sigma$-spaces. This important class of spaces recently emerged as the natural setting for a general theory of $K$-analytic sets $[\mathbf{5}, \mathbf{6}]$, and can be characterized as the images of metrizable spaces under upper semicontinuous compact-valued maps that preserve $\sigma$-discretely decomposable collections.

One possible solution to the above problem, in view of our foregoing remarks, would be to determine conditions under which all Souslin subsets of a space are generalized $F_{\sigma}$-subsets. This is in fact the approach we take, and we are led in

Received by the editors February 7, 1985 .

1980 Mathematics Subject Classification. Primary 54B99, 54D15, 54H05; Secondary 04A15, $28 \mathrm{~A} 05$.

Key words and phrases. Souslin set, generalized $F_{\sigma}$-set, covering properties, $\Sigma$-space, $P$-space, refinement $\sigma$-locally finite maps. 
a natural way to the concept of a $P$-space due to K. Morita [13] (see 2.3 for the definition). In 2.10 we prove

THEOREM 1. Let $X$ be a subparacompact $P(\omega)$-space. Then every Souslin subset of $X$ is a generalized $F_{\sigma}$-set in $X$. Consequently, if $P$ is any topological property that is hereditary with respect to generalized $F_{\sigma}$-subsets and $X$ belongs to $P$, then every Souslin subset of $X$ belongs to $P$.

By a topological space we will always mean a regular space.

In $[\mathbf{3}, \S 9]$ a study was made of topological properties inherited by all Baire subsets (i.e., members of the smallest $\sigma$-algebra containing the zero-sets) of certain topological spaces. Since all Baire sets are Souslin sets, the following result can be viewed as a complement and partial extension of Theorem 9.10 of $[\mathbf{3}]$ (see 2.12 below for the proof).

THEOREM 2. Let $X$ be a completely regular Hausdorff space and let $S$ be a Souslin subset of $X$.

(a) If $X$ is realcompact, then $S$ is realcompact.

(b) If $X$ is topologically complete, then $S$ is topologically complete (in the sense of having a complete uniformity).

(c) If $X$ is a $P(\omega)$-space and $X$ is either Lindelöf, or paracompact, or normal and subparacompact, then $S$ will be a $P(\omega)$-space having the respective property. (See also Theorem 7 below.)

Generalized $F_{\sigma}$-sets often inherit topological properties in a strong way (cf. $[\mathbf{9}, \mathrm{p}$. 836]). In particular, we say that a subspace $S$ of a topological space $X$ is subparacompact in $X$ provided any cover of $S$ by open sets in $X$ has a partial refinement by a closed and $\sigma$-discrete (equivalently, $\sigma$-locally finite) collection relative to the space $X$, and which covers $S$. (Recall that in a "partial" refinement, the union of the refining family need not be equal to the union of the original family.) In 2.2 below we prove

THEOREM 3. Let $X$ be any (regular) space. Then (a) and (b) of the following are equivalent for any subspace $S$ of $X$, and both are implied by (c).

(a) $S$ is subparacompact in $X$.

(b) $S$ is (relatively) subparacompact and a generalized $F_{\sigma}$-set in $X$.

(c) $S$ is a generalized $F_{\sigma}$-set in $X$ and $X$ is subparacompact.

Property (a) of Theorem 3 recently emerged as an important concept in a study of non-Lindelöf $K$-analytic sets (see, in particular, $\S 4$ of $[5]$ ). Its usefulness here stems from the equivalence in Theorem 3 and the following theorem on the preservation of this property (see 2.5 for the proof).

THEOREM 4. Let $F: X \rightarrow Y$ be an upper semicontinuous set-valued map with $F(x)$ a Lindelof set in the space $Y$ for each $x$ in $X$. Suppose $F$ maps closed discrete collections in $X$ to collections that have a $\sigma$-locally finite refinement in $Y$. Then, for any subspace $S$ of $X$, if $S$ is subparacompact in $X$, the image $F(S)$ is subparacompact in $Y$.

Although the full generality of Theorem 4 will be useful elsewhere, here we are primarily interested in determining conditions under which the projection map $X \times \mathbf{P} \rightarrow X$, parallel to the space $\mathbf{P}$ of irrational numbers, is index- $\sigma$-locally finite (see 2.4 for this definition), so that the conclusion of Theorem 4 will hold for this 
map. Indeed, since the Souslin subsets of a space $X$ are precisely the projections of the closed sets of $X \times \mathbf{P}$, Theorem 1 will be an immediate consequence of Theorems 3 and 4 in view of the following theorem (see 2.7 for the proof).

THEOREM 5. Let $X$ be a $P(\omega)$-space and $Y$ a Lindelöf $\Sigma$-space. Then the projection map $p: X \times Y \rightarrow X$ is index- $\sigma$-locally finite. If, in addition, $X$ is subparacompact, then $p$ maps generalized $F_{\sigma}$-sets in $X \times Y$ to generalized $F_{\sigma}$-sets in $X$.

The assumptions in Theorem 5 on the spaces $X$ and $Y$ are to a large extent also necessary for the conclusion to hold. For example, if $I$ is the unit interval and $D$ is an uncountable discrete space, then the projection map $I \times D \rightarrow I$ fails to preserve even $\sigma$-discrete sets. Thus we cannot omit the assumption that $Y$ is Lindelöf. Also, if $M$ is the "Michael line" space [10] (a hereditarily paracompact non- $P(\omega)$-space), then the projection map $M \times \mathbf{P} \rightarrow M$ is not index- $\sigma$-locally finite (see 2.8 below). In fact, we prove below in 2.9 the following partial converse of Theorem 5 .

THEOREM 6. Suppose for all subspaces $Y$ of $\mathbf{P}$ the projection map $X \times Y \rightarrow X$ is index- $\sigma$-locally finite, and $X \times Y$ is subparacompact. Then $X$ is a subparacompact $P(\omega)$-space.

As a particular consequence of the above results we state the following portmanteau theorem on the topological properties of Souslin sets in $\Sigma$-space (in particular, Souslin subsets of compact spaces crossed with metrizable spaces).

THEOREM 7. Let $X$ be a $\Sigma$-space and $S$ a Souslin subset of $X$. Then the following hold.

(a) $S$ is a $\Sigma$-space.

(b) If $X$ is completely regular Hausdorff, then $S$ is realcompact or topologically complete whenever $X$ has the respective property $(X$ need not be a $\Sigma$-space in this case).

(c) If, additionally, $X$ is subparacompact (i.e., $X$ is a strong $\Sigma$-space), then $S$ is a generalized $F_{\sigma}$-set in $X$. Consequently, in this case, if $X$ has any one of the following topological properties, then so will $S$ : Lindelöf, paracompact, normal, subparacompact, metacompact, submetacompact, countably paracompact and normal, para-Lindelöf and normal, and collectionwise normal.

REMARK. Part (a) of Theorem 7 was first shown by K. Nagami in the special case when $S$ is an $F_{\sigma \delta}$-set or when $S$ is a Borel set and $X$ is perfectly normal (cf. $3.5,3.9$, and 3.20 of $[\mathbf{1 4}])$.

Our final theorem deals with the descriptive nature of Souslin sets in $\Sigma$-spaces and, more generally, $P(\omega)$-spaces.

THEOREM 8. Let $X$ be a subparacompact $P(\omega)$-space and let $S$ be a Souslin subset of $X$.

(a) If $S$ is a $G_{\delta}$-set in $X$ [and $X$ is normal], then $S$ is an $F_{\sigma \delta}$-set in $X$ [respectively, a countable intersection of cozero-sets in $X]$.

(b) If $S$ is an open set in $X$ [and $X$ is normal], then $S$ is an $F_{\sigma}$-set in $X$ [respectively, a cozero-set in $X]$.

(c) If every open set of $X$ is a Souslin set, then $X$ is perfect (i.e., all open sets in $X$ are $F_{\sigma}$-sets). 
(d) If $X$ is normal, then the zero-sets [and hence the Baire sets] of $S$ are precisely the intersections of zero-sets [Baire sets] of $X$ with $S$; in particular, $S$ is z-embedded in $X$.

REMARK. The assumption in part (c) is sometimes made in place of the stronger assumption that the space is perfect (e.g., see Theorems 5.8.10, 5.8.11, and 5.9.2 of $[7])$. The result (c) shows that this assumed generality may often be illusory.

In a perfectly normal space, the Borel and Baire sets are the same. An old problem of M. Katetov asks whether a normal space in which the Borel and Baire sets coincide must be perfect. Part (c) of the above theorem gives an affirmative answer in the case of a subparacompact $P(\omega)$-space.

\section{Definitions and proofs.}

2.1. Generalized $F_{\sigma}$-sets. Following $[\mathbf{9}]$ we call a subset $S$ of a topological space $X$ a generalized $F_{\sigma}$-subset if, whenever $S \subset U$ and $U$ is open in $X$, then $S \subset F \subset U$ for some $F_{\sigma}$-set $F$ in $X$. This concept is due to $\mathrm{Y}$. Smirnov who used the term "normally placed set" (see [4, p. 84] for the reference and an outline of basic results). It is known that normality, collectionwise normality, and nearly all covering properties are hereditary with respect to $F_{\sigma}$-subsets (see $[\mathbf{4}$ and $\mathbf{2}$, Theorem 7.1$]$ ), and it is an easy exercise to show that this continues to hold for generalized $F_{\sigma}$-subsets. As noted in the introduction, such subsets often inherit a property in a strong way as exemplified by Theorem 3 .

2.2. Proof of TheOREM 3. Let $X$ be a (regular) space, and suppose $S$ is a generalized $F_{\sigma}$-subset of $X$ that is subparacompact in its relative topology. Let $\mathcal{U}$ be a cover of $S$ by open sets of $X$. By regularity, let $\mathcal{V}$ be a cover of $S$ by open sets of $X$ whose closures form a partial refinement of $\mathcal{U}$. Since $S$ is subparacompact, we have

$$
S=\bigcup_{n=1}^{\infty} \bigcup \nVdash(n),
$$

where each $\mathcal{H}(n)$ is a closed and discrete collection in $S$ which partially refines $\mathcal{V}$. Fix $n$, and for each $x$ in $S$ choose an open set $W(x)$ in $X$ such that $x \in W(x) \subset \bigcup \mathcal{V}$, and $W(x)$ meets at most one member of $H(n)$. Let $V(n)$ denote the union of the sets $W(x)$, for all $x$ in $S$, and note that $\mathcal{H}(n)$ is discrete relative to the subspace $V(n)$. Since $S$ is a generalized $F_{\sigma}$-set in $X$, there is an $F_{\sigma}$-set $F(n)$ such that $S \subset F(n) \subset V(n)$. Let $F(n)=\bigcup_{m=1}^{\infty} F(n, m)$, where each $F(n, m)$ is closed in $X$. It follows that

$$
\{F(n, m) \cap \bar{F}: F \text { in } \nLeftarrow(n), m=1,2, \ldots\}
$$

is a closed and $\sigma$-discrete collection in $X$ which partially refines $\mathcal{U}$. Doing this for each $n$ we obtain the desired $\sigma$-discrete partial refinement of $\mathcal{U}$, proving that $S$ is subparacompact in $X$. This proves (b) $\rightarrow$ (a).

Conversely, suppose $S$ is subparacompact in $X$ and let $V$ be an open set in $X$ containing $S$. By assumption, $\{V\}$ has a partial refinement $\not$, where $\not$ is a closed $\sigma$-discrete collection in $X$ which covers $S$. It follows that $\bigcup \mathscr{H}$ is a $F_{\sigma}$-subset of $X$ satisfying $S \subset \cup \mathcal{H} \subset V$. This proves (a) $\rightarrow$ (b).

To show that $(\mathrm{c}) \rightarrow(\mathrm{a})$, assume $(\mathrm{c})$ and let $\mathcal{U}$ be a cover of $S$ by open sets in $X$. We can choose an $F_{\sigma}$-set $F$ in $X$ such that $S \subset F \subset \cup U$. If $F=\bigcup_{n=1}^{\infty} F(n)$, where each $F(n)$ is closed in $X$, then $F(n)$ is subparacompact and so is the union 
of a $\sigma$-discrete collection of closed sets in $X$ which partially refines $U$. Doing this for each $F(n)$ provides the necessary $\sigma$-discrete partial refinement of $\mathcal{U}$.

2.3. $P$-spaces and $\Sigma$-spaces. Let $k$ be a nonzero cardinal. A space $X$ is said to be a $P(k)$-space if, given any set $A$ of cardinality $k$ and an open cover of $X$ of the form

$$
\left\{V\left(a_{1}, \ldots, a_{n}\right): a_{i} \text { in } A, n=1,2, \ldots\right\}
$$

where

$$
V\left(a_{1}, \ldots, a_{n}\right) \subset V\left(a_{1}, \ldots, a_{n}, a_{n+1}\right)
$$

whenever $a_{1}, \ldots, a_{n+1}$ belong to $A$, then there is a closed cover

$$
\left\{H\left(a_{1}, \ldots, a_{n}\right): a_{i} \text { in } A, n=1,2, \ldots\right\}
$$

of $X$ such that

(i) $H\left(a_{1}, \ldots, a_{n}\right) \subset V\left(a_{1}, \ldots, a_{n}\right)$, for all $a_{1}, \ldots, a_{n}$ in $A$, and

(ii) if $\left(a_{n}\right)$ is any sequence in $A$, then

$$
\bigcup_{n=1}^{\infty} V\left(a_{1}, \ldots, a_{n}\right)=X \text { implies } \bigcup_{n=1}^{\infty} H\left(a_{1}, \ldots, a_{n}\right)=X .
$$

A space is a $P$-space if it is a $P(k)$-space for all cardinals $k>0$. In spite of the technical difficulty in the definition, this concept (due to K. Morita [13]) is often characteristic of spaces whose products with metrizable (or $\Sigma$-) spaces have a certain topological property $(\mathrm{cf} .[\mathbf{2}, \mathbf{8}, \mathbf{1 3}])$. We will make use of the fact that, if in the definition of a $P(k)$-space we assume only that the sets $H\left(a_{1}, \ldots, a_{n}\right)$ are $F_{\sigma}$-sets in $X$ rather than closed sets, the resulting class of spaces is the same [13, Lemma 3.1]. It follows that all perfect spaces are $P$-spaces.

Another important class of $P$-spaces are the $\Sigma$-spaces first defined by K. Nagami [14]. A space $X$ is a $\Sigma$-space if there is a $\sigma$-locally finite closed collection $\mathcal{F}$ in $X$ and a cover $C$ of $X$ by closed countably compact sets such that, if $C \subset G$, where $C$ belongs to $C$ and $G$ is open in $X$, then $C \subset F \subset G$ for some $F$ in $\mathcal{F}$. If the members of $C$ are compact, then $X$ is said to be a strong $\Sigma$-space. E. Michael [11] has observed that the strong $\Sigma$-spaces coincide with the subparacompact $\Sigma$-spaces. This class of spaces forms a natural extension of both the metrizable and compact spaces.

2.4. Index- $\sigma$-locally finite and related maps. We will only summarize the definition of these maps and refer the reader to [12] for the details and background. A map $f: X \rightarrow Y$ of topological spaces (not necessarily single-valued) is said to be refinement- $\sigma$-locally finite [respectively, base- $\sigma$-locally finite] if, whenever $\mathcal{E}$ is locally finite in $X$, then $f(\mathcal{E})$ has a $\sigma$-locally finite refinement [base] in $Y$; if, in addition, $\{f(E): E$ in $\mathcal{E}\}$ is always a point-countable family, then $f$ is said to be index- $\sigma$-locally finite. The three concepts coincide if the set $\{x \in X: f(x) \cap\{y\} \neq \emptyset\}$ is a Lindelöf set in $X$ for each $y$ in $Y$ [12, Proposition 4.4]. It can be shown that a space is a subparacompact $\Sigma$-space iff $X$ is the image of a metrizable space under a compact-valued upper semicontinuous map having any one of the above three mapping properties. (If the metrizable space is also complete, we obtain the concept of a $K$-analytic space-see [5, Theorem 1].)

2.5. Proof of TheOREM 4. Suppose $F: X \rightarrow Y$ is upper semicontinuous, with $F(x)$ a Lindelöf subspace of the (regular) space $Y$ for each $x$ in $X$; and let $S$ 
be subparacompact in $X$. Let $\mathcal{U}$ be any cover of $F(S)$ by open sets of $Y$, and use regularity to obtain an open collection $\mathcal{V}$ whose closures refine $\mathcal{U}$. Let $\mathcal{V}^{*}$ denote the family of all unions of countable subcollections of $\mathcal{V}$. The assumptions on $F$ now imply that the sets $\{x \in X: F(x) \subset V\}$, for $V$ in $\mathcal{V}^{*}$, form an open cover of $S$ in $X$. Since $S$ is subparacompact in $X$, this cover of $S$ has a partial refinement of the form $\bigcup_{n=1}^{\infty} \nvdash_{n}$, where each $\nvdash_{n}$ is a closed discrete collection in $X$, and together they cover $S$. By assumption, $F\left(\mathscr{H}_{n}\right)$ has a $\sigma$-locally finite refinement in $Y$, for each $n$, and together these yield a $\sigma$-locally finite collection $\mathcal{E}$ in $Y$ which covers $F(S)$ and partially refines $\mathcal{V}^{*}$. Now for each $E$ in $\mathcal{E}$ choose a sequence $(V(E, n))$ in $\mathcal{V}$ that covers $E$, and observes that

$$
\{\bar{E} \cap \overline{V(E, n)}: E \text { in } \mathcal{E}, \text { and } n=1,2, \ldots\}
$$

will be a closed $\sigma$-locally finite family in $Y$ which covers $F(S)$ and partially refines $U$. This proves that $F(S)$ is subparacompact in $Y$.

2.6. COROLlary. Let $X$ be a subparacompact space and $F: X \rightarrow Y$ an upper semicontinuous map onto the space $Y$ with $F(x)$ Lindeloff for each $x$ in $X$. Suppose $F$ is refinement- $\sigma$-locally finite. Then:

(a) $Y$ is subparacompact, and $F$ maps generalized $F_{\sigma}$-sets in $X$ to generalized $F_{\sigma}$-sets in $Y$;

(b) if $F$ has compact values and $X$ is a $P(k)$-space, then $Y$ is a $P(k)$-space.

PROOF. Part (a) is an immediate consequence of Theorems 3 and 4. To prove (b), let $A$ be a set of cardinality $k>0$, and suppose $Y$ is covered by open sets $V(a \mid n)=V\left(a_{1}, \ldots, a_{n}\right)$ as in the definition of a $P(k)$-space (2.3). If the set of all sequences $a=\left(a_{n}\right)$ of elements in $A$ for which $Y=\bigcup_{n=1}^{\infty} V(a \mid n)$ is empty, then there is nothing more to show in this case. Otherwise, define

$$
W(a \mid n)=\{x \in X: F(x) \subset V(a \mid n)\},
$$

and note that these sets form an open cover of $X$ (some may be emtpy), with $W(a \mid n) \subset W(a \mid n+1)$ for each sequence $a=\left(a_{n}\right)$. Now let $H(a \mid n) \subset W(a \mid n)$ be closed sets in $X$ satisfying the definition of a $P(k)$-space for the open sets $W(a \mid n)$. By (a), $F(H(a \mid n))$ is a generalized $F_{\sigma}$-set in $Y$, and so there are $F_{\sigma}$-sets $K(a \mid n)$ in $Y$ such that

$$
F(H(a \mid n)) \subset K(a \mid n) \subset V(a \mid n)
$$

for each finite sequence $a \mid n=a_{1}, \ldots, a_{n}$ in $A$. It is routine to check that

$$
\bigcup_{n=1}^{\infty} V(a \mid n)=Y \text { implies } \bigcup_{n=1}^{\infty} K(a \mid n)=Y \text {. }
$$

This proves that $Y$ is a $P(k)$-space.

2.7 Proof of Theorem 5. Let $X$ be a $P(\omega)$-space, let $Y$ be a Lindelöf $\Sigma$ space, and let $p$ denote the projection map $X \times Y \rightarrow X$. Given an arbitrary locally finite family in $X \times Y$, say

$$
\{E(d): d \in D\}
$$

we need to show there exist (index) locally finite families

$$
\{E(d, n): d \in D\}
$$


in $X$, for $n=1,2, \ldots$, such that $p(E(d))$ is the union of $\{E(d, n): n=1,2, \ldots\}$ for each $d$ in $D$.

Let $\{F(1), F(2), \ldots\}$ be a sequence of closed sets in $Y$ and $C$ a cover of $Y$ by compact sets as in the definition of a Lindelöf $\Sigma$-space. Let $W$ be an open cover of $X \times Y$, closed to finite unions, such that each member of $\mathcal{W}$ meets only finitely many of the sets $E(d)$. For each finite sequence $a_{1}, \ldots, a_{n}$ of natural numbers define

$$
F(a \mid n)=\bigcap_{i=1}^{n} F\left(a_{i}\right) .
$$

$V\left(a_{i}\right)=\bigcup\left\{V: V\right.$ is open in $X$ and $V \times F\left(a_{i}\right) \subset W$ for some $\left.W \in \mathcal{W}\right\}$,

and

$$
V(a \mid n)=\bigcup_{i=1}^{n} V\left(a_{i}\right)
$$

The family of open sets $V(a \mid n)$ is easily seen to be an open cover of $X$ with $V(a \mid n) \subset$ $V(a \mid n+1)$, for each $a \mid n$, and so there exist corresponding closed sets $H(a \mid n)$ which cover $X$ and satisfy the definition of a $P(\omega)$-space (2.3).

For each $a \mid n$ we define

$$
E(d, a \mid n)=p[(H(a \mid n) \times F(a \mid n)) \cap E(d)],
$$

for each $d$ in $D$, and proceed to show that

$$
p[E(d)]=\bigcup_{a \mid n} E(d, a \mid n),
$$

where the union is over all $a \mid n$, and also that

$$
\{E(d, a \mid n): d \text { in } D\} \text { is locally finite in } \mathrm{X},
$$

for each $a \mid n$. Since the set of all such finite sequences $a \mid n$ is countable, this will complete the proof of Theorem 5 .

To prove (i), suppose $\left(x^{\prime}, y^{\prime}\right)$ belongs to $E(d)$, for a given $d$, and let $C^{\prime}$ be some member of $C$ containing $y^{\prime}$. Let $a^{\prime}$ denote the sequence

$$
\left\{a_{n}^{\prime}: n=1,2, \ldots\right\}=\left\{a: C^{\prime} \subset F(a)\right\} .
$$

For any $x$ in $X$, since $\mathcal{W}$ is an open cover of $X \times Y$ closed to finite unions, we have $\{x\} \times C^{\prime} \subset W$, for some $W$ in $\mathcal{W}$, and so

$$
\{x\} \times C^{\prime} \subset U \times V \subset W
$$

for open sets $U \supset\{x\}$ and $V \supset C^{\prime}$. Thus, for some $a_{n}^{\prime}$, we have $C^{\prime} \subset F\left(a_{n}^{\prime}\right) \subset V$, and so $x$ belongs to $V\left(a^{\prime} \mid n\right)$. This shows that $X$ is the union of the sets $V\left(a^{\prime} \mid n\right)$, and hence also the sets $H\left(a^{\prime} \mid n\right)$, for $n=1,2, \ldots$ It follows that $\left(x^{\prime}, y^{\prime}\right)$ belongs to $H\left(a^{\prime} \mid n\right) \times F\left(a^{\prime} \mid n\right)$, for some $n$, proving that $x^{\prime}$ belongs to $E\left(d, a^{\prime} \mid n\right)$. Since the reverse inclusion is obvious, that completes the proof of (i).

To prove (ii) it suffices to show that the family $\{E(d, a \mid n): d$ in $D\}$ is locally finite relative to each of the closed subspaces $H(a \mid n)$ of $X$. Fix $a \mid n$ and $x$ in $H(a \mid n)$. Since $H(a \mid n)$ is contained in $V(a \mid n), x$ has an open neighborhood $V$ such that $V \times F(a \mid n) \subset W$, for some $W$ in $\mathcal{W}$. Now it is easy to check that if $V$ meets $E(d, a \mid n)$, then $V \times F(a \mid n)$ will meet $E(d)$. As $W$ can meet $E(d)$ for only a finite 
number of indices $d$, it follows that $V$ can meet $E(d, a \mid n)$ for at most finitely many $d$.

Finally, suppose, in addition, $X$ is a subparacompact space. By a theorem of D. J. Lutzer [8], the product space $X \times Y$ is subparacompact. Thus, if $S$ is a generalized $F_{\sigma}$-set in $X \times Y$, then $S$ is subparacompact in $X \times Y$ by Theorem 3 , hence $p(S)$ is subparacompact in $X$ by Theorem 4 , and so $p(S)$ is a generalized $F_{\sigma}$-set in $X$, by a second application of Theorem 3 .

2.8. EXAMPLE. As observed in the introduction, the assumption that $Y$ is Lindelöf in Theorem 5 cannot be omitted (even when $Y$ is discrete and $X=[0,1]$ ). As for the assumptions on $X$, let $M$ be the set of real numbers topologized so that the irrationals are discrete and the rationals have their usual neighborhoods. This space is hereditarily paracompact and Hausdorff $[\mathbf{1 0}]$, and the product of $M$ with the space $\mathbf{P}$ of irrational numbers is hereditarily both subparacompact and metacompact [2, Example 6.4]; but $M \times \mathbf{P}$ is not normal [10]. Now every subset of $M$ is an $F_{\sigma \delta}$-set, as is easy to see, and so is the image of a closed set of $M \times \mathbf{P}$ under the projection map (since this is true of any Souslin subset). If this projection map were index- $\sigma$-locally finite, each subset of $M$ would be a generalized $F_{\sigma}$-set in $X$ (by 2.6 above). In particular, the open set of irrational points would be an $F_{\sigma}$-set in $M$. This implies that the set of rational points is a $G_{\delta}$-set in $M$, and hence also in the reals, but this would contradict the Baire category theorem.

2.9. Proof of ThEOREM 6. Suppose $X$ is a space such that, for all $Y \subset \mathbf{P}$, $X \times Y$ is subparacompact, and the projection $p: X \times Y \rightarrow X$ is index- $\sigma$-locally finite. For each finite sequence $a \mid n=a_{1}, \ldots, a_{n}$ of natural numbers let $V(a \mid n)$ be an open set in $X$ such that the collection $\{V(a \mid n)\}$ covers $X$ as in the definition of a $P(\omega)$-space (2.3). Recall that the space $\mathbf{P}$ can be identified with the product of countably many copies of the space $\mathbf{N}$ of natural numbers, and we assume that this identification has been made. Thus let $Y$ be the set of all points $a=\left(a_{n}\right)$ in $\mathbf{P}$ such that $X$ is the union of the sets $V(a \mid n)$, for $n=1,2, \ldots$. Let $Y(a \mid n)$ denote the set of all points $b$ in $Y$ such that $b|n=a| n$, and note that these sets form a countable open base for $Y$. It follows that the collection

$$
\{V(a \mid n) \times Y(a \mid n): a \text { in } Y \text { and } n=1,2, \ldots\}
$$

is an open cover of the subparacompact space $X \times Y$. Accordingly, by taking unions of members of a $\sigma$-discrete closed refinement of this cover, we can obtain a family of $F_{\sigma}$-sets $F(a \mid n)$ which cover $X \times Y$ and satisfy

$$
F(a \mid n) \subset V(a \mid n) \subset Y(a \mid n)
$$

for each $a \mid n$. By Theorems 3 and $4, p[F(a \mid n)]$ is a generalized $F_{\sigma}$-set in $X$; thus we can find an $F_{\sigma}$-set $H(a \mid n)$ in $X$ such that

$$
p[F(a \mid n)] \subset H(a \mid n) \subset V(a \mid n)
$$

for each $a \mid n$. Clearly, the sets $H(a \mid n)$ cover $X$. Moreover, for any $x$ in $X$ and $a$ in $Y,(x, a)$ belongs to some $F(b \mid n)$ (since these cover $X \times Y$ ), and this implies that $a|n=b| n$, since $a$ must belong to $Y(b \mid n)$; consequently, $x$ is in $p[F(a \mid n)]$, proving that $X$ is the union of the sets $H(a \mid n)(n=1,2, \ldots)$ for each $a$ in $Y$. This proves that $X$ is a $P(\omega)$-space. Since $X \times Y$ is a subparacompact space, $X$ is also subparacompact.

The above results now make it easy to prove Theorem 1. 
2.10. Proof OF TheOREM 1. Let $X$ be a subparacompact $P(\omega)$-space. By Theorem 5 , the projection map onto $X$ parallel to the space $\mathbf{P}$ of irrational numbers takes generalized $F_{\sigma}$-sets in $X \times \mathbf{P}$ to generalized $F_{\sigma}$-sets in $X$. Since $S$ is a Souslin set in $X$ if, and only if, $S$ is the projection of a closed set in $X \times \mathbf{P}$ (see, e.g., [7, $2.6]$ ), it follows that every Souslin set in $X$ is a generalized $F_{\sigma}$-set.

2.11. Proof OF THEOREM 7. (a) Let $S$ be a Souslin subset of the $\Sigma$-space $X$. Since $\Sigma$-spaces are $P$-spaces [14, Theorem 2.7], the projection map $X \times \mathbf{P} \rightarrow X$ will be index- $\sigma$-locally finite by Theorem 5 , hence so will the restriction of this map to any closed subspace of $X \times \mathbf{P}$. It follows that $S$ is a continuous index- $\sigma$-locally finite image of a $\Sigma$-space, and thus (as is easily shown) is itself a $\Sigma$-space (cf. [12, Theorem 8.1]). The same proof shows that a Souslin subset of a strong $\Sigma$-space is a strong $\Sigma$-space, since $X \times \mathbf{P}$ will be a strong $\Sigma$-space by [8, Proposition 2.5].

(b) Suppose $S$ is a Souslin subset of a realcompact space $X$ (completely regular Hausdorff). If $X^{\prime}$ is a compactification of $X$, then $S$ has the form $S^{\prime} \cap X$ for some Souslin subset $S^{\prime}$ of $X^{\prime}$. Since $S^{\prime}$ is Lindelöf [3, Lemma 9.10], $S^{\prime}$ is realcompact, and so $S$ is realcompact as the intersection of two realcompact subspaces of $X^{\prime}$ (cf. the proof of Theorem 9.10 of $[\mathbf{3}]$ ). The same proof works for the propety of topological completeness.

(c) That each of the properties listed is hereditary with respect to $F_{\sigma}$-subsets is known (see, e.g., [4 and 2, Theorem 7.1]). It is an easy exercise to show that these properties are also hereditary with respect to generalized $F_{\sigma}$-subsets $(\mathbf{c f}$. [9, p. 836]). If $X$ is any subparacompact $P(\omega)$-space, the conclusion now follows from Theorem 1.

2.12. PROOF OF THEOREM 2. This clearly follows from 2.11 and 2.6 part (b).

2.13. Proof OF TheOREM 8. Let $X$ be a subparacompact $P(\omega)$-space, and let $S$ be a Souslin subset of $X$. By Theorem $1, S$ is a generalized $F_{\sigma}$-set in $X$.

(a) If $S=\bigcap G(n)(n=1,2, \ldots)$, where each $G(n)$ is open in $X$, then there are $F_{\sigma}$-sets $F(n)$ satisfying $S \subset F(n) \subset G(n)$, and it follows that $S$ is an $F_{\sigma \delta}$-set in $X$. If $X$ is also normal, the sets $F(n)$ can be chosen to be open $F_{\sigma}$-sets [4, p. 84], and thus $S$ will be a countable intersection of cozero-sets.

(b) This is clear from the proof of (a).

(c) This follows immediately from (b).

(d) This follows from the fact that a generalized $F_{\sigma}$-set $S$ in a normal space $X$ is $z$-embedded in $X[\mathbf{1}$, Theorem 7.13], that is, the zero-sets of $S$ are precisely the sets of the form $Z \cap S$ where $Z$ is a zero-set in $X$. The corresponding property for all Baire sets now follows.

2.14. EXAMPLES. We conclude by indicating how standard examples can be used to show that the properties listed in part (c) of Theorem 7 are not in general hereditary with respect to $F_{\sigma \delta}$-subsets.

Let $P$ denote the class of all regular spaces having a given property of the type mentioned. If $X$ belongs to $P$ and $Y$ is $\sigma$-compact, then $X \times Y$ belongs to $P[\mathbf{2}$, Theorem 6.1]. Consequently, if $X$ belongs to $P$ but $X \times \mathbf{P}$ does not, then $X \times \mathbf{P}$ is an $F_{\sigma \delta}$-subset of $X \times \mathbf{R}$ of the desired type (where $\mathbf{P}$ is the space of irrationals and $\mathbf{R}$ the space of reals). For example, if $M$ is the space described in 2.8 above, then $M \times \mathbf{P}$ is a nonnormal $F_{\sigma \delta}$-subset of the paracompact space $M \times \mathbf{R}$. 


\section{REFERENCES}

1. R. A. Alo and H. L. Shapiro, Normal topological spaces, Cambridge Univ. Press, Cambridge, 1974 .

2. D. Burke, Covering properties, Handbook of Set-Theoretic Topology, North-Holland, Amsterdam, 1984.

3. W. W. Comfort and S. Negrepontis, Continuous pseudometrics, Lecture Notes in Pure and Appl. Math., Vol. 14, Dekker, New York, 1975.

4. R. Engelking, General Tpology, Polish Scientific Publishers, Warsaw, 1977.

5. R. W. Hansell, J. E. Jayne and C. A. Rogers, K-analytic sets, Mathematika 30 (1983), $189-221$.

6. R. W. Hansell, J. E. Jayne and C. A. Rogers, Separation of K-analytic sets, Mathematika (to appear).

7. J. E. Jayne and C. A. Rogers, K-analytic sets, Analytic Sets, Academic Press, London, 1980.

8. D. J. Lutzer, Another property of the Sorgenfrey line, Compositio Math. 24 (1972), 359363.

9. E. Michael, A note on paracompact spaces, Proc. Amer. Math. Soc. 4 (1953), 831-838.

10. _ The product of a normal space and a metric space need not be normal, Bull. Amer. Math. Soc. 69 (1963), 375-376.

11. __ On Nagami's $\Sigma$-spaces and some related matters, Proc. Washington State Univ. Conf. on General Topology, Pullman, Wash., 1970, pp. 13-19.

12. $\ldots$, On maps related to $\sigma$-locally finite and $\sigma$-discrete collections of sets, Pacific J. Math. 98 (1982), 139-152.

13. K. Morita, Products of normal spaces with metric spaces, Math. Ann. 154 (1964), 365-382.

14. K. Nagami, $\Sigma$-spaces, Fund. Math. 65 (1969), 169-192.

Department of Mathematics, University of Connecticut, Storrs, ConnecTICUT 06268 